\title{
Ovarian Sclerosing Stromal Tumor
}

National Cancer Institute

\section{Source}

National Cancer Institute. Ovarian Sclerosing Stromal Tumor. NCI Thesaurus. Code C4204.

A benign ovarian stromal tumor characterized by the presence of cellular areas which contain fibroblasts and round cells. The cellular areas are separated by sclerotic or edematous hypocellular tissue. Symptoms include abdominal discomfort and menstrual abnormalities. 